S. Das · M. Shahmanesh • M. Stolinski • F. Shojaee-Moradie · W. Jefferson •

N. C. Jackson • M. Cobbold · P. Nightingale · A. M. Umpleby

\title{
In treatment-naïve and antiretroviral-treated subjects with HIV, reduced plasma adiponectin is associated with a reduced fractional clearance rate of VLDL, IDL and LDL apolipoprotein B-100
}

Received: 25 July 2005 / Accepted: 2 October 2005 / Published online: 24 January 2006

(C) Springer-Verlag 2006

\begin{abstract}
Aims/hypothesis: We hypothesised that loss of peripheral fat in HIV patients would result in decreased plasma adipocytokines, in particular adiponectin, and that this decrease would be associated with changes in VLDL, IDL and LDL apolipoprotein B kinetics. Methods: Plasma adiponectin, leptin and other cytokines were measured in uninfected control subjects $(n=12)$ and three HIV-positive groups comprising treatment-naïve patients $(n=15)$ and patients on triple antiretroviral therapy containing protease inhibitors (PI, $n=15$ ) or non-nucleoside reverse transcriptase inhibitors (NNRTI, $n=25$ ). VLDL, IDL and LDL apolipoprotein B kinetics were measured with an infusion of $\left[1-{ }^{13} \mathrm{C}\right]$ leucine. Regional body fat
\end{abstract}

S. Das $(\bowtie) \cdot$ M. Shahmanesh

Departments of HIV, Chemical Pathology and Nuclear

Medicine, University Hospitals Birmingham,

Birmingham, UK

e-mail: satyajit.das@coventrypct.nhs.uk

Tel.: +44-2476224-055

Fax: +44-2476844-199

M. Stolinski · F. Shojaee-Moradie - W. Jefferson ·

N. C. Jackson - A. M. Umpleby

Department of Endocrinology and Diabetes,

St Thomas' Hospital, GKT School of Medicine,

London, UK

M. Cobbold

Department of Immunology,

University of Birmingham Medical School,

Birmingham, UK

P. Nightingale

Wellcome Trust Clinical Research Facility,

Queen Elizabeth Hospital,

Birmingham, UK

S. Das

Department of GU Medicine, University Hospitals Coventry and Warwickshire, Stoney Stanton Road,

Coventry CV1 4FH, UK was measured with a dual energy X-ray absorptiometry scan. Insulin resistance was calculated using homeostasis model assessment (HOMA). Results: Adiponectin (median [interquartile range]) was reduced in the treatmentnaive $(5.4 \mu \mathrm{g} / \mathrm{ml}[4.7-8.5])$, PI $(5.0 \mu \mathrm{g} / \mathrm{ml}[3.3-6.4])$ and NNRTI $(5.0 \mu \mathrm{g} / \mathrm{ml}$ [3.1-6.7]) groups compared with controls $(9.7 \mu \mathrm{g} / \mathrm{ml}[6.9-13.3])(p<0.05)$. In all subjects adiponectin correlated positively with HDL-cholesterol levels, the VLDL, IDL and LDL apolipoprotein B fractional clearance rates, and with the limb fat:lean body mass ratio (all $p<0.01$ ). Adiponectin correlated negatively with plasma triglyceride levels and HOMA $(p<0.001)$. In a linear regression model that included HOMA, adiponectin was an independent predictor of VLDL and HDL-cholesterol levels and the IDL fractional clearance rate. TNF was higher in treatment-naive and PI subjects, and soluble TNF receptor superfamily, members $1 \mathrm{~A}$ and 1B (previously known as TNF receptors 1 and 2) was higher in PI patients than in control subjects $(p<0.05)$. Conclusions/interpretation: Adiponectin levels are significantly reduced in treated and untreated HIV patients and are predictive of VLDL and IDL apolipoprotein B fractional clearance rates. Adiponectin may have a direct effect on lipoprotein metabolism, which may be independent of insulin.

Keywords Adiponectin - Antiretroviral treatment . Cytokines - Dyslipidaemia - Human immunodeficiency virus · IDL apolipoprotein B kinetics · LDL apolipoprotein B kinetics $\cdot$ Lipodystrophy $\cdot$ VLDL apolipoprotein B kinetics

Abbreviations ApoB: Apolipoprotein B100 - ASR: Absolute secretion rate FCR: Fractional clearance rate HOMA: Homeostasis model assessment - LBM: Lean body mass · NNRTI: Non-nucleoside reverse transcriptase inhibitors - PI: protease inhibitors . TNFRSF1A: TNF receptor superfamily, member 1A; TNFRSF1B, TNF receptor superfamily, member 1B 


\section{Introduction}

The adipocyte is an active endocrine secretory cell producing several cytokines with possible important metabolic regulatory activities, both locally and at other body sites [1]. Loss of peripheral fat may alter the secretion of adipocytokines with consequent metabolic effects [2]. The most abundant gene transcript within the adipocyte is adiponectin, plasma concentrations of which correlate strongly with insulin sensitivity [3]. The presence of different adiponectinsensitive receptors in liver and skeletal muscles has been demonstrated [4], suggesting adiponectin may have a role in controlling metabolism in these tissues. Hypertriglyceridaemia and low HDL-cholesterol concentrations in women are associated with low adiponectin concentrations [5], while in obese subjects a correlation between adiponectin and VLDL apolipoprotein B-100 (apoB) fractional clearance rate (FCR) has been demonstrated [6]. In mice administration of adiponectin has been shown to reduce dyslipidaemia induced by the protease inhibitor ritonavir [7].

We recently demonstrated in HIV patients a significant reduction in VLDL, IDL and LDL apoB fractional catabolic rates $[8,9]$, which was inversely related to peripheral fat. The underlying cause for the altered lipid metabolism and consequent dyslipidaemia may be loss of peripheral fat, but the mechanism is not understood.

\section{Subjects and methods}

\section{Subjects}

We performed a cross-sectional study on 67 subjects who were HIV-positive $(n=55)$ or presumed HIV-negative (control subjects; $n=12$ ). The HIV-positive subjects were either treatment-naïve $(n=15)$ or had been taking two nucleoside analogues plus either a protease inhibitor (PI, $n=15$ ) or a non-nucleoside reverse transcriptase inhibitor (NNRTI, $n=25$ ) for between 1 and 6 years. None of the subjects was infected with hepatitis $\mathrm{C}$ virus. Exclusion criteria were: fasting glucose $>6.0 \mathrm{mmol} / \mathrm{l}$; treatment with glucocorticoids and/or any drugs that affect lipid metab- olism; hypothyroidism; creatinine $>150 \mathrm{mmol} / \mathrm{l}$; alanine aminotransferase and aspartate aminotransferase $>$ five times upper limit of normal; anaemia; and $>10 \%$ loss in body weight in the preceding 6 months. The study had ethical committee approval and all subjects gave written informed consent.

\section{Experimental protocol}

VLDL, IDL and LDL enrichment, apoB concentration and lipid composition were measured following an infusion of $\left[1-{ }^{13} \mathrm{C}\right]$ leucine after an overnight fast as described previously $[8,9]$. Body fat distribution was measured by whole-body dual energy X-ray absorptiometry scan and expressed in terms of lean body mass (LBM). Only four patients had clinical evidence of lipodystrophy, one on PI, three on NNRTI, and none in the treatment-naive group. Four had received statins (two each in the PI and NNRTI groups), which were stopped 6 weeks before testing, so at the time of testing subjects were not on lipid-lowering drugs.

\section{Assay methods}

The enrichment and concentration of VLDL, IDL and LDL apoB, the lipid composition of VLDL, IDL and LDL, and plasma concentrations of NEFA, insulin and glucose were measured as described previously [8, 9]. Plasma adiponectin and leptin were measured by radioimmunoassay kits (Linco Research, St Louis, MO, USA). Cytokine levels were measured in a multiplex bead sandwich immunoassay (Bioscource International, Camarillo, CA, USA) on a Luminex 100 (Bio-Rad Laboratories, Hemel Hempstead, UK), according to the manufacturer's instructions.

\section{Data analysis}

Insulin resistance was calculated by the homeostasis model assessment $\left(\mathrm{HOMA}_{\mathrm{IR}}\right)$. The FCR and absolute secretion

Table 1 Adipocytokines, HOMA and body fat in HIV-negative patients (controls), treatment-naïve patients (TN) and patients on highly active antiviral therapy containing protease inhibitors (PI) and non-nucleoside reverse transcriptase inhibitors (NNRTI)

\begin{tabular}{lccccc}
\hline & Control & TN & PI & NNRTI & $p$ value between groups \\
\hline Adiponectin $(\mu \mathrm{g} / \mathrm{ml})$ & $9.7(6.9-13.3)$ & $5.4^{\mathrm{a}}(4.7-8.5)$ & $5.0^{\mathrm{a}}(3.3-6.4)$ & $5.0^{\mathrm{a}}(3.1-6.7)$ & 0.003 \\
HOMA $_{\mathrm{IR}}$ & $0.9(0.6-1.3)$ & $1.3(0.9-1.9)$ & $1.4(1.0-3.3)$ & $1.3(0.5-2.0)$ & NS \\
Leptin $(\mathrm{ng} / \mathrm{ml})$ & $8.7(3.4-13.6)$ & $4.4(2.5-7.5)$ & $4.2(3.3-6.2)$ & $2.1^{\mathrm{a}}(1.1-4.9)$ & 0.006 \\
TNF $(\mathrm{pg} / \mathrm{ml})$ & $0.75(0.5-0.98)$ & $3.7^{\mathrm{a}, \mathrm{b}}(2.6-4.2)$ & $1.5^{\mathrm{a}}(1.3-4.7)$ & $1.1(1.0-2.1)$ & 0.001 \\
TNFRSF1A (pg/ml) & $412(272-674)$ & $598(326-793)$ & $831^{\mathrm{a}}(650-947)$ & $670(523-842)$ & 0.03 \\
TNFRSF1B (pg/ml) & $1044(845-1214)$ & $1331^{\mathrm{a}}(1143-1720)$ & $1699^{\mathrm{a}}(1102-2053)$ & $1449^{\mathrm{a}}(1120-1633)$ & 0.015 \\
IL8 (pg/ml) & $4.0(3.3-4.2)$ & $6.0(4.2-7.5)$ & $5.8(4.7-6.4)$ & $4.9(3.9-7.6)$ & NS \\
Limb fat/LBM & $0.21(0.14-0.29)$ & $0.15(0.09-0.22)$ & $0.09^{\mathrm{a}}(0.07-0.15)$ & $0.08^{\mathrm{a}}(0.06-0.14)$ & $<0.001$ \\
Trunk fat/LBM & $0.16(0.11-0.21)$ & $0.16(0.11-0.19)$ & $0.13(0.10-0.19)$ & $0.13(0.08-0.20)$ & NS \\
\hline
\end{tabular}

Values are medians (interquartile ranges)

${ }^{\mathrm{a}} p<0.05$ vs controls; ${ }^{\mathrm{b}} p<0.05$ vs NNRTI 
rate (ASR) of VLDL, IDL and LDL were calculated as previously described $[8,9]$. Statistical analysis was by Kruskal-Wallis followed by Dunn's multiple comparison tests (SPSS 10.0.7). Correlations were by Spearman's rank correlation test. In a stepwise forward linear regression model, we examined the effect of adiponectin, leptin, TNF IL8, TNF receptor superfamily members $1 \mathrm{~A}$ and $1 \mathrm{~B}$ (TNFRSF1A, TNFRSF1B, previously known as TNFR-1 and TNFR-2), HOMA, NEFA, age, ethnicity, sex, alcohol intake, smoking, and family history of cardiovascular disease or diabetes on VLDL, IDL and LDL metabolism and fasting plasma lipids. Non-normal data were log transformed.

\section{Results}

Subject demographics, and VLDL, IDL and LDL kinetics and composition have been previously described $[8,9]$. Limb fat was significantly lower in the two treatment groups, while trunk fat was similar among all the groups (Table 1). Adiponectin was lower in all HIV patients, including treatment-naive patients, than in control subjects $(p<0.05)$, but was not different between the three HIV groups (Table 1). Leptin levels were lower in the NNRTI group than in control subjects $(p<0.05)$. TNFRSF1A and TNFR SF1B were higher in PI-treated patients than in control subjects. TNFRSF1B was also higher in TN and NNRTI-treated patients than in control subjects $(p<0.05$, Table 1). TNF was higher in treatment-naive patients than in control subjects and in the NNRTI group $(p<0.05)$ and also higher in the PI group than in control subjects $(p<0.05)$. IL1 $\beta$, IL6, IL10 and IL12p70 are not shown as they were below the detection limit of the assay for most samples.

There was a negative correlation of adiponectin with HOMA $\left(r_{\mathrm{s}}=-0.43, p<0.001\right)$ and positive correlation with limb fat $/$ LBM $\left(r_{\mathrm{s}}=0.6, p<0.001\right.$, Fig. 1a) but not with trunk fat/LBM. Leptin correlated with total fat/LBM $\left(r_{\mathrm{s}}=0.87\right.$, $p<0.001)$ and limb fat $/ \mathrm{LBM}\left(r_{\mathrm{s}}=0.79, p<0.001\right)$. HOMA correlated with trunk fat $/ \mathrm{LBM}\left(r_{\mathrm{s}}=0.39, p=0.002\right)$ but not with limb fat/LBM or total fat/LBM. Adiponectin was lower in patients with dyslipidaemia (cholesterol $\geq 6 \mathrm{mmol} / 1$ and/or triglyceride $\geq 2.3 \mathrm{mmol} / \mathrm{l}$ ) than in those without $(3.3 \mu \mathrm{g} / \mathrm{ml}$ [2.4-5.1] $(n=14)$ vs $5.4 \mu \mathrm{g} / \mathrm{ml}$ [4.7-7.9], $p=0.01, n=40$ ), but there was no significant difference in leptin, HOMA or regional body fat changes.

Adiponectin correlated positively with HDL-cholesterol $\left(r_{\mathrm{s}}=0.57, p<0.001\right)$, VLDL apoB FCR $\left(r_{\mathrm{s}}=0.47, p<0.001\right.$, Fig. 1b), IDL apoB FCR $\left(r_{\mathrm{s}}=0.5, p<0.001\right.$, Fig. $\left.1 \mathrm{c}\right)$, LDL apoB FCR $\left(r_{\mathrm{s}}=0.36, p<0.004\right)$, and IDL apoB ASR $\left(r_{\mathrm{s}}=0.35, p=0.008\right)$. It correlated negatively with total triglycerides $\left(r_{\mathrm{s}}=-0.53, p<0.001\right)$, VLDL, IDL and LDL apoB pool size $\left(r_{\mathrm{s}}=-0.46,-0.31\right.$ and $-0.43, p<0.001$, $p=0.012$ and $p<0.001$, respectively), VLDL, IDL and LDLcholesterol $\left(r_{\mathrm{s}}=-0.5,-0.44\right.$ and $-0.26, p<0.001, p<0.001$ and $p=0.02$, respectively), VLDL triglyceride $\left(r_{\mathrm{s}}=-0.48\right.$, $p<0.001)$, and soluble TNFRSF1A $\left(r_{\mathrm{s}}=-0.25, p=0.04\right)$. HOMA correlated with VLDL, IDL and LDL apoB FCR $(r=-0.32,-0.33$ and $-0.42, p=0.01, p=0.01$ and $p=0.001$, respectively). There was no correlation between the baseline HIV viral load, the CD4 count and the VLDL, IDL or LDL apoB FCR.

In a linear regression model, adiponectin predicted VLDL and IDL apoB FCR $(p=0.012, p<0.001$, respectively) and HDL-cholesterol $(p<0.001)$, and negatively predicted VLDL triglyceride $(p=0.004)$, IDL-cholesterol: apoB ratio $(p=0.025)$, and total triglyceride $(p<0.001)$. HOMA predicted LDL apoB pool size $(p=0.04)$ and total triglyceride $(p=0.001)$ and negatively predicted IDL and LDL ASR ( $p=0.02$ and $p=0.006$, respectively). Leptin predicted VLDL-cholesterol $(p<0.001)$ and IDL-cholesterol: apoB ratio $(p<0.001)$. Soluble TNFRSF1A predicted VLDL and IDL apoB concentration $(p=0.017$ and $p=0.007$, respectively), IDL-cholesterol $(p=0.001)$, VLDL and IDL
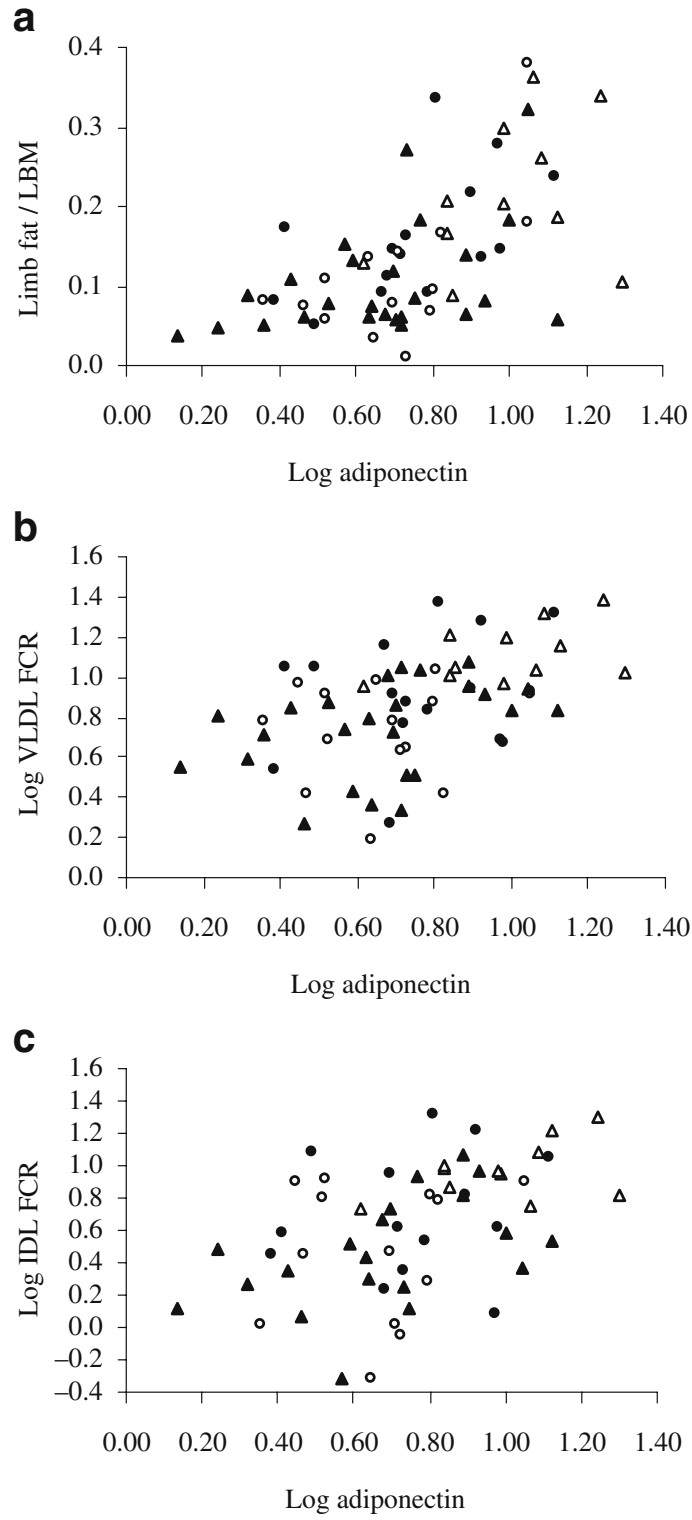

Fig. 1 Correlation of plasma adiponectin with (a) peripheral fat/LBM $\left(r_{\mathrm{s}}=0.6, p<0.001\right),(\mathbf{b}) \operatorname{VLDL}$ FCR $\left(r_{\mathrm{s}}=0.47, p<0.001\right)$ and (c) IDL FCR $\left(r_{\mathrm{s}}=0.5, p<0.001\right)$. Controls (closed triangles), treatment-naive (solid circles), PI (open triangles), NNRTI (open circles) 
apoB pool size ( $p=0.028$ and $p=0.007$, respectively), as well as total cholesterol and triglyceride $(p=0.001)$. It negatively predicted VLDL and LDL FCR and LDL ASR ( $p=0.01$, $p<0.001$ and $p=0.03$, respectively). Soluble TNFRSF1B predicted VLDL-cholesterol ( $p=0.03)$, LDL-cholesterol and triglyceride levels $(p=0.002$ and $p=0.025$, respectively), as well as the LDL apoB pool size and concentration $(p<0.001$ and $p=0.001$, respectively). It negatively predicted the LDL FCR $(p=0.004)$. TNF negatively predicted HDL-cholesterol $(p=0.002)$. IL8 predicted LDL triglyceride $(p<0.025)$.

\section{Discussion}

This study shows that, in comparison with HIV-negative control subjects, both HIV-infected patients taking antiretroviral medication containing PI or NNRTI and treatment-naïve HIV patients show a significant reduction in adiponectin. A correlation was found between adiponectin and limb fat loss and also with VLDL, IDL and LDL apoB FCR. Although adiponectin levels correlated inversely with insulin resistance as measured by HOMA, adiponectin in a linear regression model that included HOMA was an independent predictor of VLDL and IDL FCR, and of HDL-cholesterol levels. Interestingly, soluble TNFRSF1A also predicted VLDL FCR, apoB pool size and concentration, as well as total cholesterol and triglyceride, while TNFRSF1B predicted LDL FCR, LDL apoB pool size and concentration, plus cholesterol and triglyceride content.

The decrease in adiponectin in HIV patients in the present study is consistent with previous studies of HIV patients with lipodystrophy [3], although to our knowledge reduced adiponectin levels have not been previously reported in treatment-naïve HIV patients. Interestingly, there was no evidence of lipodystrophy in the treatmentnaïve group. It has been suggested that low adiponectin levels may lead to insulin resistance and that insulin resistance may cause the abnormalities in lipid metabolism in HIV lipodystrophy [3]. However, we were unable to demonstrate a significant change in insulin resistance, as measured by HOMA, in any of the treatment groups. Moreover, although HOMA correlated with IDL and LDL apoB ASR, it did not correlate with VLDL or IDL apoB FCR, even when adiponectin was removed from the regression model. Leptin levels, which correlated closely with limb and trunk fat/LBM, did not correlate with VLDL, IDL or LDL apoB FCR. Adiponectin levels were significantly lower in the dyslipidaemic patients than in patients with normal lipid levels, whereas HOMA and leptin levels were not different. Our results suggest that adiponectin could play a role in VLDL, IDL and LDL metabolism, and that its role could be independent of insulin action.

Adiponectin levels were associated with a loss of limb fat, while insulin resistance was associated with an increase in trunk fat. Atrophic adipose tissue contains poorly developed adipocytes and a marked alteration in gene expression [10]. It is likely that lipoatrophy leads to reduced gene expression of adiponectin. Since VLDL, IDL and LDL apoB FCRs correlate with limb fat, this suggests that the primary abnormality is lipodystrophy. In a previous study from Sutinen et al. [11], lipodystrophic HIV patients had peripheral lipoatrophy, but more intra-abdominal fat and liver fat than HIV patients without lipodystrophy and than HIV-negative controls, while BMI remained the same. A direct negative correlation between hepatic steatosis and plasma adiponectin concentration was shown in another study [12]. We are unable to comment on intra-abdominal fat accumulation in our study, as this was not measured separately. It is possible that low adiponectin may lead to increased liver fat and that this may affect VLDL and IDL FCRs. Reduced plasma adiponectin may lead to reduced clearance of VLDL and IDL apoB by inhibiting lipoprotein lipase and hepatic lipase. Lipoprotein lipase mRNA has also been shown to be lower in HIV patients with lipodystrophy than in patients without lipodystrophy [13], suggesting that regulation may be at the level of transcription. In a transgenic mouse with chronically elevated adiponectin, lipoprotein lipase has been shown to be increased [14]. The prolonged residence time of apoBcontaining lipoproteins will allow exchange of triglyceride for cholesterol esters with HDL. This would increase clearance, providing a mechanism for the reduced HDL-cholesterol seen in both treatment-naïve patients and in those on antiretroviral therapy, thus possibly explaining the strong correlation between adiponectin with HDL-cholesterol.

Acknowledgements This research was supported by a grant from the British Heart Foundation (PG/2001/153) and the Wellcome Trust (064571). We are grateful to P. Croos for her technical assistance, R. Cramb for performing the insulin assays and G. Gilleran for helping to recruit patients.

\section{References}

1. Trayhurn P, Beattie JH (2001) Physiological role of adipose tissue: white adipose tissue as an endocrine and secretory organ. (Review). Proc Nutr Soc 60:329-339

2. Schmitz M, Michl G, Walli R et al (2001) Alterations of apolipoprotein B metabolism in HIV-infected patients with antiretroviral combination therapy. J Acquir Immune Defic Syndr 26:225-235

3. Addy CL, Gavrila A, Tsiodras S, Brodovicz K, Karchmer AW, Mantzoros CS (2003) Hypoadiponectinemia is associated with insulin resistance, hypertriglyceridemia, and fat redistribution in human immunodeficiency virus-infected patients treated with highly active antiretroviral therapy. J Clin Endocrinol Metab 88:627-636

4. Yamauchi T, Kamon J, Ito $\mathrm{Y}$ et al (2003) Cloning of adiponectin receptors that mediate antidiabetic metabolic effects. Nature 423:762-769

5. Matsubara M, Maruoka S, Katayose S (2002) Decreased plasma adiponectin concentrations in women with dyslipidemia. J Clin Endocrinol Metab 87:2764-2769

6. Ng TW, Watts GF, Farvid MS, Chan DC, Barrett PH (2005) Adipocytokines and VLDL metabolism: independent regulatory effects of adiponectin, insulin resistance, and fat compartments on VLDL apolipoprotein B-100 kinetics? Diabetes $54: 795-802$

7. Xu A, Yin S, Wong L, Chan KW, Lam KS (2004) Adiponectin ameliorates dyslipidemia induced by the human immunodeficiency virus protease inhibitor ritonavir in mice. Endocrinology 145:487-494 
8. Shahmanesh M, Das S, Stolinski M et al (2005) Antiretroviral treatment reduces VLDL and IDL apolipoprotein B fractional catabolic rate in HIV infected patients with mild dyslipidaemia J Clin Endocrinol Metab 90:755-760

9. Umpleby AM, Das S, Stolinski M et al (2005) LDL apolipoprotein B metabolism in treatment naïve HIV patients and patients on antiretroviral therapy. Antivir Ther 10:663-670

10. Kannisto K, Sutinen J, Korsheninnikova E et al (2003) Expression of adipogenic transcription factors, peroxisome proliferator-activated receptor gamma co-activator 1, IL-6 and CD45 in subcutaneous adipose tissue in lipodystrophy associated with highly active antiretroviral therapy. AIDS 17:1753-1762

11. Sutinen J, Hakkinen AM, Westerbacka J et al (2002) Increased fat accumulation in the liver in HIV-infected patients with antiretroviral therapy-associated lipodystrophy. AIDS 16:2183-2193
12. Bugianesi E, Pagotto U, Manini R et al (2005) Plasma adiponectin in nonalcoholic fatty liver is related to hepatic insulin resistance and hepatic fat content, not to liver disease severity. J Clin Endocrinol Metab 90:3498-3504

13. Bastard JP, Caron M, Vidal H et al (2002) Association between altered expression of adipogenic factor SREBP1 in lipoatrophic adipose tissue from HIV-1-infected patients and abnormal adipocyte differentiation and insulin resistance. Lancet 359:1026-103

14. von Eynatten M, Schneider JG, Humpert PM et al (2004) Decreased plasma lipoprotein lipase in hypoadiponectinemia: an association independent of systemic inflammation and insulin resistance. Diabetes Care 27:2925-2929 